\title{
Elevated macrophage-inducible C-type lectin expression in the synovial tissue of patients with rheumatoid arthritis
}

\author{
KEN TAKATA, SHOTARO TAKANO, MASAYUKI MIYAGI, MANABU MUKAI, DAI IWASE, \\ JUN AIKAWA, YOSHIHISA OHASHI, GEN INOUE, MASASHI TAKASO, KENTARO UCHIDA \\ Department of Orthopedic Surgery, Kitasato University School of Medicine, 1-15-1 Minami-ku Kitasato, Sagamihara City, Kanagawa, Japan
}

\begin{abstract}
Rheumatoid arthritis (RA), a systemic autoimmune disease, is known to cause chronic inflammation in synovial joints. A number of inflammatory conditions are associated with stimulation of Clec4e, a macrophage-inducible C-type lectin (MINCLE) and transmembrane pattern recognition receptor that functions in innate immunity. We previously reported MINCLE expression in synovial macrophages isolated from the synovium of osteoarthritis $(O A)$ patients. However, MINCLE expression has not been examined in RA synovial tissue. To examine MINCLE expression in RA patients, synovial tissue specimens were obtained from patients with $R A$ and OA during joint replacement surgery $(n=20$ each). Total RNA was extracted from synovial tissue and used to compare MINCLE expression in OA and $R A(n=15$ each). We also extracted fresh CD14+ (macrophage-rich) and CD14-cell fractions from synovial tissue and compared MINCLE expression between $O A$ and RA patients $(n=5$ each). MINCLE levels in synovial tissue were significantly elevated in RA patients compared to OA patients. MINCLE expression was significantly elevated in the CD14+ fraction compared to the CD14-fraction in both $O A$ and $R A$ patients. Further, while there were no differences in the CD14+fraction between $R A$ and $O A, M I N C L E$ expression in the CD14-fraction was elevated in RA compared to OA. Our findings indicate that MINCLE expression is elevated in the synovium of RA patients and that MINCLE expression in non-macrophage cell fractions may be a key feature of $R A$.
\end{abstract}

Key words: rheumatoid arthritis, synovial tissue, MINCLE.

(Cent Eur J Immunol 2021; 46 (4): 470-473)

\section{Introduction}

Rheumatoid arthritis (RA), a systemic autoimmune disease, is known to cause chronic systemic manifestations and accompanying synovitis, which can lead to joint damage and disability. Given that RA has a heterogeneous phenotype and is associated with several pathogenic pathways, identification of the molecules responsible for RA pathogenesis is key for developing effective therapeutic strategies.

Monocytes/macrophages play a key role in RA progression by generating inflammatory factors within the inflamed synovium [1]. In addition, monocytes/macrophages are also central to pathological bone erosion in RA owing to the high rate at which they differentiate into osteoclasts [2]. $\mathrm{Clec} 4 \mathrm{e}$ is a macrophage-inducible C-type lectin (MINCLE) and a transmembrane pattern recognition receptor that functions in innate immunity [3, 4]. MINCLE is predominantly expressed in monocytes/macrophages and is stimulated in several inflammatory conditions [5-10]. Nakamura et al. reported augmented MINCLE mRNA levels in bone marrow-derived mononuclear cells (BMMC) taken from patients with RA compared to osteoarthritis (OA) [10]. We previously reported MINCLE expression in synovial macrophages isolated from the synovium of OA patients, confirming that MINCLE levels may be elevated in inflammatory conditions [9]. A recent study also reported that MINCLE contributes to osteoclastogenesis [11]. Therefore, elevated MINCLE levels may contribute to the pathogenesis of inflammation and bone destruction in RA. However, MINCLE expression has not been examined in the synovial tissue (ST) of RA patients.

Here, we examined MINCLE expression in ST from RA and OA patients.

\section{Material and methods}

\section{Patients}

ST specimens were obtained from 20 RA and 20 OA patients during joint replacement surgery. All RA patients

Correspondence: Kentaro Uchida, Department of Orthopedic Surgery, Kitasato University School of Medicine, 1-15-1 Minami-ku Kitasato, Sagamihara City, Kanagawa 252-0374, Japan, e-mail: kuchida@med.kitasato-u.ac.jp Submitted: 11.03.2021; Accepted: 22.10.2021 
satisfied the 2010 American College of Rheumatology/ European League Against Rheumatism (ACR/EULAR) criteria [12]. Meanwhile, the OA patients were all radiographically diagnosed with unilateral Kellgren/Lawrence $(\mathrm{K} / \mathrm{L})$ grades 3 and $4 \mathrm{OA}$. This study protocol received approval from the Ethics Review Board of Kitasato University (reference number: KMEO 19-259).

Fifteen of the 20 specimens from each group were immediately subjected to analysis of MINCLE expression using quantitative polymerase chain reaction (qPCR), while the remaining five specimens were used to isolate macrophages.

\section{Comparison of MINCLE expression in ST}

Methods used for total RNA extraction from ST and isolation of macrophage cells and cDNA synthesis were based on previous studies $[9,13]$. PCR primer pair sequences used for qPCR analysis were: MINCLE-sense (5'-GTG CCT GTT TCA TCA CCA GA-3') and MINCLE-antisense (5'-TTC CCA GTT CAA TGG ACA ACA A-3') for MINCLE amplification (product size: $152 \mathrm{bp}$ ); $C D 14$-sense (5'-TCC CTC AAT CTG TCG TTC GC-3') and CD14-antisense (5'-ATT CCC GTC CAG TGT CAG GT-3') for CD14 amplification (product size: $150 \mathrm{bp}$ ); and $G A P D H$-sense (5'-TGT TGC CAT CAA TGA CCC CTT-3) and GAPDHantisense (5'-CTC CAC GAC GTA CTC AGC G-3') for GAPDH amplification (product size: $202 \mathrm{bp}$ ). Melting curve analysis was used to examine qPCR product specificity. Relative MINCLE, CD14, GAPDH mRNA levels were determined using qPCR [9]. MINCLE and CD14 mRNA levels were normalized to that of the housekeeping gene $G A P D H$.

\section{Isolation of CD14+ and CD14- cells}

To examine MINCLE expression in macrophages, we extracted synovial macrophages from the ST of the knees of $5 \mathrm{OA}$ and 5 RA patients. ST was digested with collagenase and the resulting cells were incubated with biotin-conjugated mouse anti-human CD14 monoclonal antibody (1:20; clone M5E2, BioLegend) for $30 \mathrm{~min}$ utes at $4^{\circ} \mathrm{C}$. After washing twice with phosphate-buffered saline (PBS), the cells were added to streptavidin-conjugated magnetic particles (BD Biosciences, CA, USA) and separated in a magnetic separation system (BD IMag cell separation system, BD Biosciences) into CD14+ (mac- rophage) and CD14- cells, as described elsewhere [13]. Freshly extracted CD14+ and CD14- cell fractions were subjected to qPCR to analyze CD14 and MINCLE expression in OA and RA.

\section{Statistical analysis}

Normal distribution of data was confirmed using the Kolmogorov-Smirnov test, and differences in CD14 and MINCLE expression between RA and OA groups were compared using the Mann-Whitney $U$ test. Differences in MINCLE expression between CD14- and CD14+ cells were determined using the paired $t$-test or paired Wilcoxontest. Comparisons among 3 or more groups were conducted using the Kruskal-Wallis test. $p<0.05$ was used to indicate statistical significance. All statistical analyses were performed using SPSS software (v. 25.0; IBM Corp., Armonk, NY, USA).

\section{Results \\ MINCLE expression in ST of OA and RA patients}

Patients in the OA and RA groups had comparable demographic characteristics, including age, male/female ratio, and body mass index (Table 1). CD14 expression was likewise similar in OA and RA patients $(p=0.561$; Fig. 1A). In contrast, MINCLE expression was significantly elevated in ST from RA compared to OA patients $(p=0.005$; Fig. 1B).

\section{MINCLE expression in CD14+ and CD14- fractions}

To identify the synovial cells expressing MINCLE, we examined MINCLE expression in CD14+ and CD14- cell fractions. To confirm that CD14+ cells had been successfully isolated, we also examined $C D 14$ expression. In both the OA and RA groups, $C D 14$ expression was significantly higher in the CD14+ cell fraction ( $p=0.032$ and $p=0.015$, respectively; Fig. 2A). Similarly, MINCLE expression was significantly elevated in the CD14+ cell fraction in both the OA and RA groups ( $p=0.043$ and $p=0.043$, respectively; Fig. 2B). Further, while no significant differences were observed in the CD14+ fraction between RA and OA

Table 1. Patients' demographic characteristics

\begin{tabular}{|c|c|c|c|c|c|c|}
\hline & \multicolumn{3}{|c|}{$\begin{array}{l}\text { Patients whose synovial tissue was analyzed } \\
\qquad(n=15)\end{array}$} & \multicolumn{3}{|c|}{$\begin{array}{l}\text { Patients whose CD14- and CD14+ cells were analyzed } \\
\qquad(n=5)\end{array}$} \\
\hline & $\mathbf{O A}$ & $\mathbf{R A}$ & $P$ value & $\mathbf{O A}$ & $\mathbf{R A}$ & $P$ value \\
\hline Age (years) & $70.9 \pm 1.9$ & $64.5 \pm 2.8$ & 0.118 & $69.2 \pm 3.3$ & $73 \pm 3.1$ & 0.465 \\
\hline Male/female & $6 / 9$ & $3 / 12$ & 0.427 & $1 / 4$ & $1 / 4$ & 1.000 \\
\hline BMI $\left(\mathrm{kg} / \mathrm{m}^{2}\right)$ & $27.0 \pm 0.9$ & $26.7 \pm 1.9$ & 0.184 & $28.9 \pm 2.8$ & $23.4 \pm 1.4$ & 0.117 \\
\hline
\end{tabular}



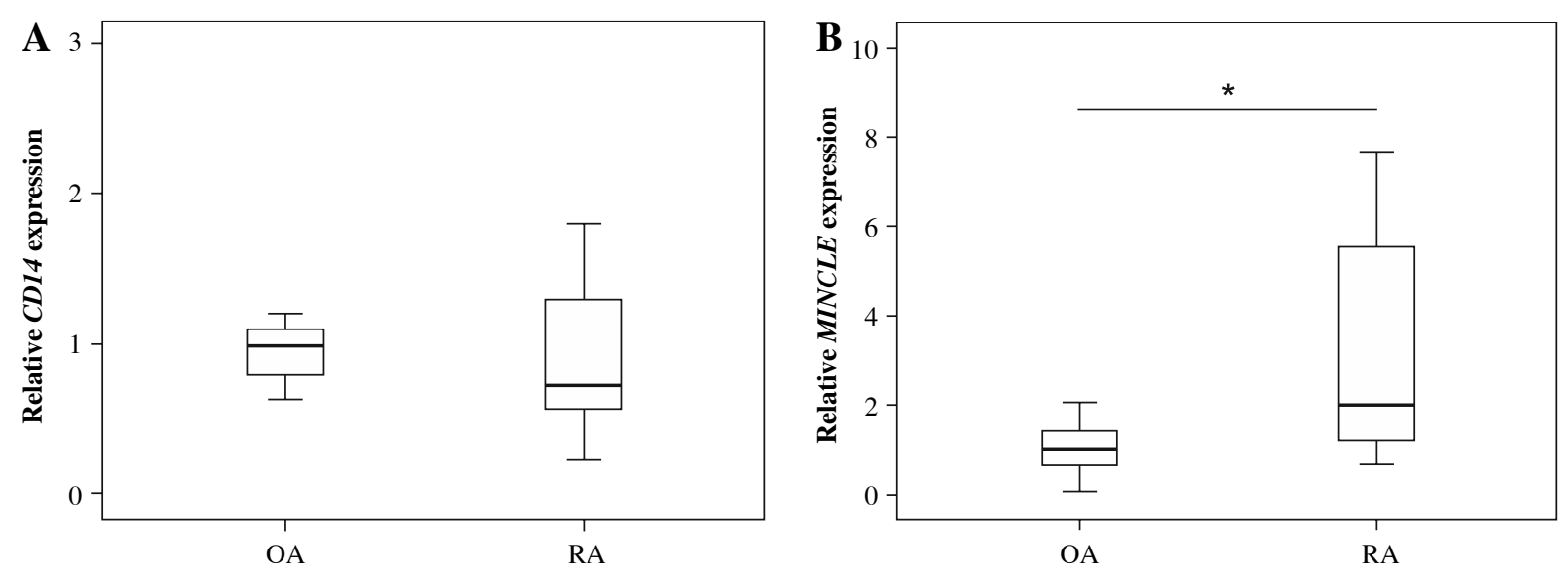

Fig. 1. MINCLE expression in synovial tissue. A) CD14 and B) MINCLE expression in synovium taken from osteoarthritis (OA) and rheumatoid arthritis (RA) patients. ${ }^{*} p<0.05$
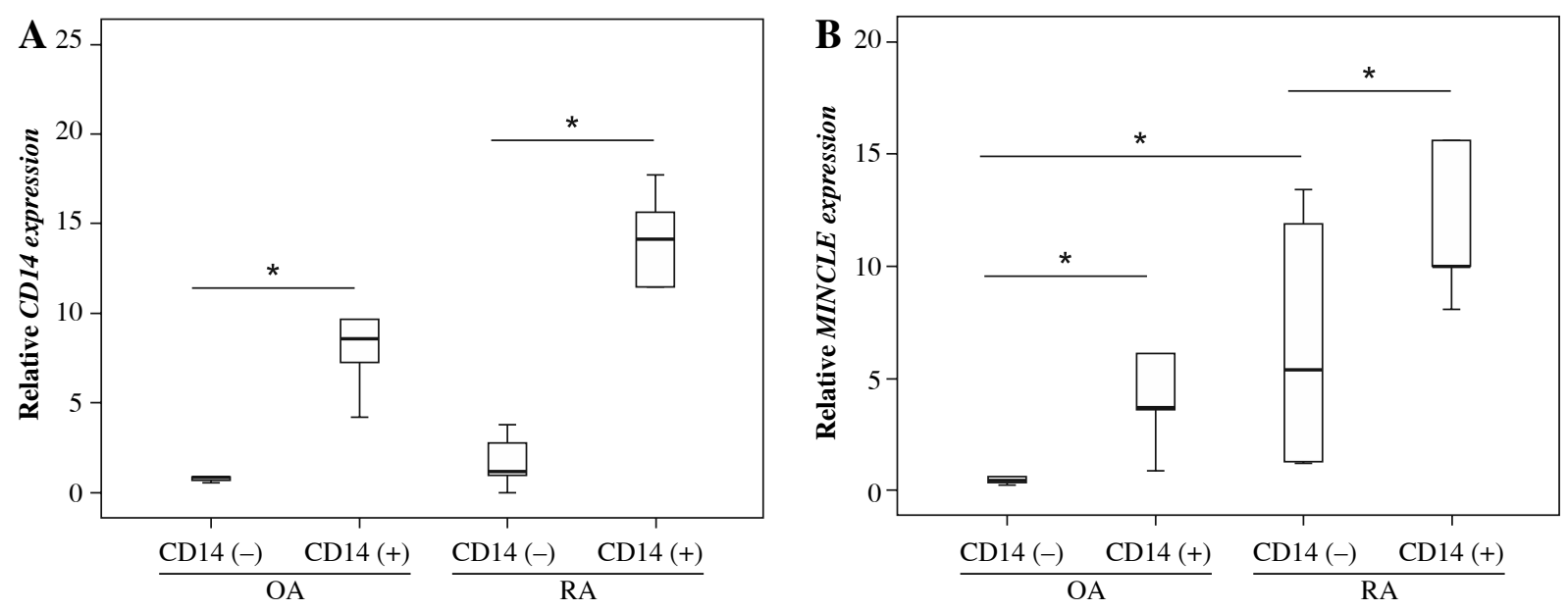

Fig. 2. CD14 and MINCLE mRNA expression in CD14- and CD14+ synovial cells. A) CD14 and B) MINCLE expression in CD14+ and CD14- cells isolated from the synovium of osteoarthritis (OA) and rheumatoid arthritis (RA) patients. $* p<0.05$

groups, MINCLE expression was significantly elevated in CD14- fractions isolated from RA compared to OA patients $(p=0.028$; Fig. $2 \mathrm{~B})$.

\section{Discussion}

Evidence suggests that MINCLE has crucial contributions to inflammation. For example, specific expression of MINCLE is observed in infiltrating M1 macrophages in the obstructed kidney of the unilateral ureteral obstruction mouse model of renal injury [8]. Further, Tanaka et al. demonstrated that the sensing of renal tubular cell death by MINCLE leads it to induce persistent inflammation following acute kidney injury in mice [14]. MINCLE has also been observed to have roles in neuroinflammation in cerebral ischemia and traumatic brain injury in rats $[15,16]$.
Here, we observed elevated synovial expression of MINCLE in RA patients. A previous study showed that injection of a MINCLE agonist induced skin inflammation in mice. Further, a MINCLE agonist induced tumor necrosis factor $\alpha$ (TNF- $\alpha$ ) production in human blood monocyte-derived macrophages, which was completely suppressed by an anti-human MINCLE antibody [17]. Together, this evidence suggests that MINCLE may be a major contributor to synovial inflammation in RA.

Damage-associated molecular patterns (DAMPs) are a group of molecules that are excreted by damaged or dying cells. When released, DAMPS activate the innate immune system and have been linked to RA $[18,19]$. A recent study showed that MINCLE senses osteocyte-released DAMPs and functions to enhance osteoclastogenesis [11]. Therefore, elevated levels of MINCLE in RA may play 
a role in bone destruction by facilitating osteoclastogenesis.

A large number of studies have reported MINCLE expression in myeloid cells [20, 21]. However, studies have also reported MINCLE expression in endothelial and neuronal cells in ischemic stroke models and stroke patients $[22,23]$. In our study, we observed elevated MINCLE expression in the CD14+ macrophage cell fraction isolated from both OA and RA patients, with no significant differences in MINCLE expression between the groups. In contrast, MINCLE expression in CD14- cells was elevated in RA compared to OA patients. These findings suggest that MINCLE expression in the non-macrophage fraction may be a key feature of RA.

Our study has a number of limitations. First, we did not examine a healthy control group. Second, all samples were extracted from patients' knees during joint replacement surgery; thus, the joints were severely damaged by the disease. Further investigation in patients with early disease is needed to reveal the importance of MINCLE in RA. Third, we did not examine the mechanism by which MINCLE contributes to RA pathology. Finally, we did not identify which cells within the CD14- fraction express MINCLE. Additional studies are needed to clarify these issues.

The authors declare no conflict of interest.

\section{References}

1. Davignon JL, Hayder M, Baron M, et al. (2013): Targeting monocytes/macrophages in the treatment of rheumatoid arthritis. Rheumatology (Oxford) 52: 590-598.

2. Takayanagi H (2007): Osteoimmunology: shared mechanisms and crosstalk between the immune and bone systems. Nat Rev Immunol 7: 292-304.

3. Richardson MB, Williams SJ (2014): MCL and Mincle: C-type lectin receptors that sense damaged self and pathogen-associated molecular patterns. Front Immunol 5: 288.

4. Yamasaki S, Ishikawa E, Sakuma M, et al. (2008): Mincle is an ITAM-coupled activating receptor that senses damaged cells. Nat Immunol 9: 1179-1188.

5. Behler F, Steinwede K, Balboa L, et al. (2012): Role of Mincle in alveolar macrophage-dependent innate immunity against mycobacterial infections in mice. J Immunol 189: 3121-3129.

6. Ichioka M, Suganami T, Tsuda N, et al. (2011): Increased expression of macrophage-inducible C-type lectin in adipose tissue of obese mice and humans. Diabetes 60: 819-826.

7. Inoue T (2017): M1 macrophage triggered by Mincle leads to a deterioration of acute kidney injury. Kidney Int 91: 526-529.

8. Lv LL, Tang PM, Li CJ, et al. (2017): The pattern recognition receptor, Mincle, is essential for maintaining the M1 macrophage phenotype in acute renal inflammation. Kidney Int 91: 587-602.

9. Moriya M, Uchida K, Takano S, et al. (2020): Expression and regulation of macrophageinducible C-type lectin in human synovial macrophages. Cent Eur J Immunol 454: 377-391.

10. Nakamura N, Shimaoka Y, Tougan T, et al. (2006): Isolation and expression profiling of genes upregulated in bone marrow-derived mononuclear cells of rheumatoid arthritis patients. DNA Res 13: 169-183.

11. Andreev D, Liu M, Weidner D, et al. (2020): Osteocyte necrosis triggers osteoclast-mediated bone loss through macrophage-inducible C-type lectin. J Clin Invest 130: 4811-4830.

12. Aletaha D, Neogi T, Silman AJ, et al. (2010): 2010 Rheumatoid arthritis classification criteria: an American College of Rheumatology/European League Against Rheumatism collaborative initiative. Arthritis Rheum 62: 2569-2581.

13. Takano S, Uchida K, Inoue G, et al. (2017): Nerve growth factor regulation and production by macrophages in osteoarthritic synovium. Clin Exp Immunol 190: 235-243.

14. Tanaka M, Saka-Tanaka M, Ochi K, et al. (2020): C-type lectin Mincle mediates cell death-triggered inflammation in acute kidney injury. J Exp Med 217: e20192230.

15. Chen S, Ma Q, Krafft PR, et al. (2013): P2X7R/cryopyrin inflammasome axis inhibition reduces neuroinflammation after SAH. Neurobiol Dis 58: 296-307.

16. He Y, Xu L, Li B, et al. (2015): Macrophage-inducible C-type lectin/spleen tyrosine kinase signaling pathway contributes to neuroinflammation after subarachnoid hemorrhage in rats. Stroke 46: 2277-2286.

17. Hattori Y, Morita D, Fujiwara N, et al. (2014): Glycerol monomycolate is a novel ligand for the human, but not mouse macrophage inducible C-type lectin, Mincle. J Biol Chem 289: $15405-15412$.

18. Roh JS, Sohn DH (2018): Damage-associated molecular patterns in inflammatory diseases. Immune Netw 18: e27.

19. McInnes IB, Schett G (2011): The pathogenesis of rheumatoid arthritis. N Engl J Med 365: 2205-2219.

20. Kawata K, Illarionov P, Yang GX, et al. (2012): Mincle and human B cell function. J Autoimmun 39: 315-322.

21. Matsumoto M, Tanaka T, Kaisho T, et al. (1999): A novel LPS-inducible C-type lectin is a transcriptional target of NF-IL6 in macrophages. J Immunol 163: 5039-5048.

22. Suzuki Y, Nakano Y, Mishiro K, et al. (2013): Involvement of Mincle and Syk in the changes to innate immunity after ischemic stroke. Sci Rep 3: 3177.

23. de Rivero Vaccari JC, Brand FJ, 3rd, Berti AF, et al. (2015): Mincle signaling in the innate immune response after traumatic brain injury. J Neurotrauma 32: 228-236. 